\title{
The Level of Copper in Hair Androgenic Alopecia
}

\section{Wiwiek Amriyana Saputri ${ }^{1}$, Farida Tabri ${ }^{1}$, Nurelly Noro Waspodo ${ }^{1}$, Burhanuddin Bahar ${ }^{2}$, Agussalim Bukhari ${ }^{3}$, Nursiah La Nafie ${ }^{4}$}

${ }^{1}$ Department of Dermatovenereology, Medical Faculty, Hasanuddin University, Makassar, Indonesia

${ }^{2}$ Biostatistic, Medical Faculty, Hasanuddin University, Makassar, Indonesia

${ }^{3}$ Department of Clinical Nutritional, Medical Faculty, Hasanuddin University, Makassar, Indonesia

${ }^{4}$ Department of Chemical, Mathematics and Sciences Faculty, Hasanuddin University, Makassar, Indonesia

\section{Email address:}

wiwiekamriyana@yahoo.com (W. A. Saputri)

\section{To cite this article:}

Wiwiek Amriyana Saputri, Farida Tabri, Nurelly Noro Waspodo, Burhanuddin Bahar, Agussalim Bukhari, Nursiah La Nafie. The Level of Copper in Hair Androgenic Alopecia. American Journal of Clinical and Experimental Medicine. Vol. 4, No. 4, 2016 , pp. 98-102. doi: 10.11648/j.ajcem.20160404.12

Received: May 20, 2016; Accepted: May 31, 2016; Published: June 17, 2016

\begin{abstract}
Androgenic alopecia is characterized by progressive loss of hair from the scalp. This research aimed to determine the hair and blood copper levels in men with androgenic alopecia. The research was conducted in the Department of Dermatovenereology of Dr. Wahidin Sudirohusodo Hospital, Makassar and the Center for Health Laboratory, Makassar, using the observational research method. The samples comprised 21 the males with androgenic alopecia and 11 control samples without androgenic alopecia. The hair and blood of the samples were analyzed using the atomic absorption spectrophotometer receipts. The research results indicated resources that the hair copper level in the androgenic alopecia group Showed a difference compared to the hair copper levels in the control group, though the difference was significant $(p<0.05)$. On the other hand, the blood copper levels in androgenic alopecia group had no difference compared to that in the control group. The hair copper levels had no correlation with the blood copper levels. The age had a significant correlation with the androgenic alopecia, in that, the alopecia incidence would increase of as the age increased $(p<0.05)$. However, the levels of both the hair and blood coppers had no correlation with the age. Neither, did the levels of both copper hair and blood have a correlation with the degree of androgenic alopecia (Hamilton grade).
\end{abstract}

Keywords: Androgenic Alopecia, Copper, Hair Loss

\section{Preliminary}

Androgenic alopecia is a form of hair loss is most often in men, but can also occur in women, involving the loss of real hair, pigmented terminal hair on the head, which responds to circulating androgens. Other names are male pattern baldness, the common hair loss, male pattern alopecia, androgen-dependent alopecia, androgenic alopecia, or baldness. [1]

Although there are no precise statistics, the incidence of Caucasians recorded close to $100 \%$, other opinions mentioned most in both men and women over 40 years showed androgenic alopecia. [1] Prevalence of androgenic alopecia (AGA) increases with age. Androgenic alopecia in women is estimated at about 21 million women around the world and is more common in older women, 6-12\% in men aged 20-30 years and increased by $40-45 \%$ over the age of 70 years. [2] There are variations in terms of race. The reason of variation in terms of race is not yet clear, but it is likely due to genetic factors as differences in appearance regardless of the location. [2, 3]

Androgenic alopecia is common and not life-threatening and is not painful but it is troublesome diseases. In the cultural orientation in industrialized countries, relationships bald with aging has a negative connotation and is often the cause of androgenic alopecia which is characterized by psychological distress and reduced quality of life for men and women. The patient feels ashamed, a feeling of being old, 
and a loss of confidence. [4]

The psychological impact of this condition affects the attractiveness of personal and social life. A study conducted in both sexes has confirmed that the AGA is a stressful condition which affects the function of individual psychological. Although in many developing countries androgenic alopecia when not accompanied by abnormal health status, is seen as a cosmetic problem, especially in males, but the psychological impact is not diminished. This is seen as a cosmetic issue perhaps because compared to many other issues are more pressing (e.g. socioeconomic). [3]

Previous research by Amirnia et al (2013), who hypothesized that decreased levels of copper and zinc causes hair loss in these patients. The concentration of elements of hair is also important. Besides the existing article, the hypothesis that the elements copper and zinc deficiency on the hair can cause the occurrence of androgenic alopecia. There is no significant difference in the levels of zinc, iron, and copper serum between patients with alopecia and normal people. He reported only universalis alopecia with urinary copper levels were significantly lower. [5]

The hair follicle is a unique structure that has an incredible ability with the potential for regeneration. It is very important for epidermal homeostasis and reepithelialization after damage to human skin. Like the others, the organ system that is more active and quick to breed, hair follicles can be easily distracted in a normal growth cycle by the effects of local and systemic. As an example of the influence of Dihydrotestosterone (DHT), a molecule derived from the action of the enzyme 5-alpha reductase (type 1 and 2) on testosterone. DHT among others cause damage to the hair follicle that leads to an inflammatory reaction to the scalp and hair follicle miniaturization development. This may be a receptor pathway abnormalities that arise. The receptor problem is probably in the fact that many subjects using chemical elements that reduce levels of DHT to occur alopecia, this may indicate involvement / disorder biochemical components in addition to testosterone and its derivatives in the pathogenesis of alopecia. [1]

Until now, the location of androgen action on hair follicles is not yet clear, but new research shows that the papillary dermis as a hair growth. Is currently thought that the differences between men and women with androgenic alopecia is associated with differences in the effect of androgens. Previously, androgenic alopecia in women is believed to be associated with increased levels of DHT which has the same mechanism in men, but the current study showed that unlike in men, women with AGA marked increase in the amount of testosterone production. [2]

There are still very few studies evaluating the androgenic alopecia and hair copper levels and some research results are still at odds about the copper content of the hair on the androgenic alopecia is the reason we investigate further. Therefore, this study aimed to evaluate the hair and blood copper levels in men with androgenic alopecia.

\section{Materials and Methods}

\subsection{Location and Time Research}

The study was conducted at the Health Sciences Dermatology, Department of Dermatovenereology Dr. Wahidin Sudirohusodo Hospital and other hospitals. Examination conducted at the Center for Health Laboratory Makassar conducted in February-March 2016.

\subsection{Design and Research Variables}

This study design was observational study to determine levels of copper hair and blood on androgenic alopecia than the control. The research variables consist of: the independent variable (copper content), the dependent variable (androgenic alopecia), variable between (hair follicles), and the control variables (gender and age).

\subsection{Population and Sample}

The study population was all androgenic alopecia patients who visited the clinic Cosmetic Dermatology Division of department of dermatovenereology, Wahidin Sudirohusodo hospital in Makassar aged 20-60 years. Samples are affordable throughout the population who meet the inclusion criteria, willing to participate in the study by signing an informed consent. Selection of research sample conducted in consecutive sampling is appropriate sampling the patient's arrival.

\subsection{Method of Collecting Data}

This research was conducted with the stages of activities as follows: 1) Preparation, 2) Explanation and signing informed consent, 3) Mechanical execution, 4) examination to assess patients androgenic alopecia using criteria Norwood-Hamilton, 5) examination to assess the levels of copper hair and serum androgenic alopecia patients by using Atomic Absorption Spectrometry (AAS), 6) procedure of specimen collection.

\subsection{Data Analysis Technique}

To know the difference between the levels of copper hair in androgenic alopecia and control, we used independent $t$ test test when the data were normally distributed copper levels; if not normal to use the Mann Whitney $U$ test. To determine the relationship (correlation) between the copper content of the hair with androgenic alopecia. Limit of significance used was $\alpha=5 \%$. The results of the analysis are collected in the form of a narrative, incorporating tables or graphs.

\section{Results}

We have conducted an observational study to evaluate the hair and blood copper levels in men with androgenic alopecia. The study was conducted at the Health Sciences Dermatology, department of Dermatovenereology, Dr. 
Wahidin Sudirohusodo hospital. Examination conducted at the Center for Health Laboratory Makassar conducted in February-March 2016. Samples taken in the form of hair on the head portion of the occipital and blood taken from $V$. The median cubital for patients suffering from androgenic alopecia and who do not suffer from androgenic alopecia.

The number of samples in this study were 33 people consisting of 22 men suffering from androgenic alopecia and 11 men who did not androgenic alopecia. In the age group suffering from androgenic alopecia between 20-60 years old on average at the age of 39 years, while the average control at the age of 30 years. Hair copper levels between androgenic alopecia i.e. (276.05) and control (151.09) but not significantly different. On blood copper levels not found differences between the groups androgenic alopecia (49.36) and the controls (49.90) (Appendix, Table 1).

Copper hair is not associated with blood copper, groups, and age ( $p>0.05)$, as well as with unrelated blood copper with copper hair, and age groups $(p>0.05)$. However, age significantly associated with androgenic alopecia ( $p$ $<0.05$ ), the older age increases the incidence of alopecia (Appendix, Table 2).

The relationship between copper hair, blood copper and degrees of androgenic alopecia (Hamilton grade) obtained a copper content of copper hair and blood is not related to the degree of androgenic alopecia (Hamilton grade) obtained $\mathrm{p}>$ 0.05 (Appendix, Table 3).

Table 1. Characteristics of Sample.

\begin{tabular}{lllll}
\hline variable & Group & N & mean & The standard deviation of \pm \\
\hline \multirow{2}{*}{ Age } & Case & 22 & 39.22 & 7.71 \\
& Control & 11 & 30.36 & 7.08 \\
\multirow{2}{*}{ Hair Cu (g/g) } & Case & 22 & 276.05 & 677.35 \\
& Control & 11 & 151.09 & 315.02 \\
$\mathrm{Cu} \mathrm{Blood} \mathrm{(mg/L)}$ & Case & 22 & 49.36 & 18.27 \\
\hline
\end{tabular}

Table 2. Relationship Between Group androgenic alopecia, hair Copper, Copper Blood and Age.

\begin{tabular}{|c|c|c|c|c|c|c|c|c|c|}
\hline variable & $\mathbf{n}$ & $\mathbf{r}$ & Hair Cu (p-value) & $\mathbf{r}$ & Cu Blood (P-value) & $\mathbf{R}$ & Group (P-value) & $\mathbf{r}$ & Age (P-value) \\
\hline Cu Hair & 33 & & & 0.05 & 0.77 & -0.10 & 0.56 & 0.15 & 0.41 \\
\hline Cu Blood & 33 & 0.51 & 0.77 & & & 0.12 & 0.94 & -0.78 & 0.66 \\
\hline Group & 33 & -0.10 & 0.56 & 0.01 & 0.94 & & & -0.49 & 0,003 \\
\hline Age & 33 & 0.14 & 0.41 & -0.08 & 0.66 & -0.49 & 0,003 & & \\
\hline
\end{tabular}

Table 3. Relationship between Hair Copper, Copper Blood and degree of androgenic alopecia (Hamilton Grade).

\begin{tabular}{lll}
\hline variable & N & Hamilton Grade (P-value) \\
\hline Cu Hair & 22 & 0.59 \\
$\mathrm{Cu}$ Blood & 22 & 0.94 \\
\hline
\end{tabular}

* Spearman's rho

\section{Discussion}

This study shows the differences between the levels of androgenic alopecia hair copper and control but not significant $(p>0.05)$. There were no differences in blood copper levels between androgenic alopecia and control. Hair copper levels are not related to blood copper levels. Age has a significant relationship with androgenic alopecia, alopecia where the incidence increases with age $(p<0.05)$. Hair and blood copper levels are not related to age. Hair and blood copper levels are also not related to the degree of alopecia (Hamilton grade).

This study chose the hair and blood copper levels associated with androgenic alopecia, although androgenic alopecia can occur genetically. Regarding the possible causes of androgenic alopecia diverse hypotheses have been introduced, but the main cause of this disease has not been discussed in any of the hypotheses. Finding possible causes of this disease may be a reference to precaution and step therapy. Different hypotheses such as the lack of some of the elements in the blood and hair have been investigated. [6]

The mean value of the hair copper content between androgenic alopecia i.e. (276.05) and control (151.09) but no significance differences among them. However, there is no significant different between the mean blood copper of the androgenic alopecia patients and controls. These results are consistent with research which was conducted by Ozturka et al (2014), in Turkey that produced a mean value of hair copper levels differ between patients with androgenic alopecia than the control and serum copper levels were not different between the two groups. [7] The research that conducted in Iran by Amirnia et al (2013), which get the hairs on the copper content of androgenic alopecia lower than the control with $\mathrm{p}<0.005$. [6] The research that conducted by Kil et al (2013), who analyzed the concentrations of copper and zinc in the hair loss results obtained serum copper not differ significantly between groups androgenic alopecia than 
controls $(\mathrm{p}=0.975)$. [8]

The hair copper is not associated with blood copper, groups, and age ( $p>0.05)$, as well as with unrelated blood copper with copper hair, and age groups $(\mathrm{p}>0.05)$. Research conducted by Amirnia et al (2013), in androgenic alopecia patients aged 17 to 62 years found that hair copper levels lower than controls age 15 to 38 years. This study did not show the relationship of age with a copper content of hair. [6] Research by Kil et al (2013), did not show any significant correlation between copper and age. [8]

This study show that age significantly associated with androgenic alopecia $(\mathrm{p}<0.05)$, the older age increases the incidence of alopecia. Androgenic alopecia is a form of hair loss that usually occurs in adulthood. Onset based on history begins at puberty. [9] According to the age, the incidence of androgenic alopecia at the age of 30 years is around $30 \%$ and is increasing at age of 50 approximately $50 \%$, and $80 \%$ at age of 70 years. $[10,11]$

The copper hair and blood levels are not related to the degree of androgenic alopecia (Hamilton grade) obtained $\mathrm{p}>$ 0.05. Direct relationship explanation between copper content of hair, blood copper and degrees of alopecia has not been explained. Based on the severity of alopecia is judged by the presence of hair thinning that occurs at a young age, a family history of androgenic alopecia, and the pattern or distribution of thinning / hair loss then the classification of the HamiltonNorwood for men with androgenic alopecia is divided into mild (grade 1 and 2), moderate (degree 3a to 4) and weight (degree 4a to 7). [12]

In this study, we get the results of different hair copper but not significantly in androgenic alopecia patients than in controls. Various biochemical functions depend on copper such as immune activity, protein synthesis and hormone action. So that copper has a major role in the physiology and biochemistry, and his involvement in the health of individuals. However, the function of copper to the hair follicle is unknown. Only one study of hair copper levels in patients with male androgenic alopecia. Zinc levels in patients with male androgenic alopecia. They showed that the levels of zinc in the male androgenic alopecia was significantly lower in men with normal hair. The authors reported that the levels of zinc may play a role in etiopathogenesis male androgenic alopecia and its treatment. Research by Ozturka et al (2014), who reported that zinc and copper hair with lower levels of androgenic alopecia in males than in controls. The result is different probably due to genetic and environmental reasons. No significant differences in the levels of copper and zinc in serum and urine. Until now, no study has compared the levels of copper and zinc in serum and urine of male androgenic alopecia. [7] On the other hand Skalnaya study (2014), which showed a much lesser extent on the content of copper and zinc in the hair of the head occipital frontal area than in women with androgenic alopecia. Comparison of level in serum and hair have two different uses. The serum level tend to show the current status of the body, and the hair level showed that long a time limit and can be measured in years. [13] Micronutrient levels in various types of alopecia except androgenic alopecia have been reported and the results are still being debated. The results were varied in levels of copper and zinc in many studies can be explained because the foundation of the sample size, methodology and variations in population. [7]

Zinc and copper is absorbed in the small intestine through a special pathway ZNT (zinc transporter), as well by divalent cationic transporter. Therefore, we can assume that if both excessive levels may affect serum concentrations of competing with the way in which this can lead to inaccurate results. Therefore, the concentration of serum copper showed no significant difference is the amount and the ratio of patients in the control group. This is due to the absence of a direct relationship between the concentration of serum copper and hair loss where it is in line with other studies that further studies are needed. [8]

Hair copper levels were significantly higher in patients than controls. Although copper has no direct role in the formation of hair, but its role in the formation of blood vessels has been demonstrated. But at the very high levels of copper acts as an oxidant, it can interfere with various biochemical reactions important for the formation of hair through the product of oxidative process. [1]

From these results, we get the difference of different hair copper levels were higher in androgenic alopecia than the control, although the number of samples slightly. Limitations of our study because only a group of men with androgenic alopecia with a little number of samples studied.

\section{Conclusions and Recommendations}

The researchers concluded that there is a difference between the levels of androgenic alopecia hair copper and control but not significant. There were no differences in blood copper levels. between androgenic alopecia and control. Hair copper levels are not related to blood copper levels. Age has a significant relationship with androgenic alopecia, alopecia where the incidence increases with age. Hair and blood copper levels are not related to age. Hair and blood copper levels are also not related to the degree of androgenic alopecia (Hamilton grade). Researchers suggest that further research with a larger number of samples to prove the relationship between copper hair and blood to do research androgenic alopecia and androgenic alopecia copper levels in women.

\section{References}

[1] Iyanda. (2012). Serum Elements Status of Androgenetic Alopecia Subjects Exposed to Cigarette Smoke or Alcohol. JETEAS. 3.702 to 707 .

[2] Schweiger et al. (2010). Update on The Pathogenesis, Genetics and Medical Treatments of Patterned Hair Loss. JDD, 9.1412-9.

[3] Iyanda AA. Serum Elements Status of Androgenetic Alopecia Subjects Exposed to Cigarette Smoke or Alcohol. JETEAS. 2012; 3(4):702-7. 
[4] Randall. (2010). Molecular Basis of Androgenetic Alopecia. United Kingdom, Springer.

[5] Dastgheib et al. (2014). Comparison of $\mathrm{Zn}, \mathrm{Cu}$, and $\mathrm{Fe}$ Content in Hair and Serum in Alopecia Areata Patients with Normal Group. Derm Resc Pract, 1-5.

[6] Amirnia et al. (2013). Assessment of Zinc and Copper Contents in the Hair and Serum and Also superoxide dismutase, glutathione Peroxidase and Malondi Aldehyde in Serum in Androgenetic Alopecia and Alopecia Areata. Life Sci J 10, 204-209.

[7] Ozturka et al. (2014). BMI and Levels of Zinc, Copper in Hair Serum and Urine of Patients With Turkish Male Androgenetic Alopecia. Trace Element J Med Biol, 28, 266-270.

[8] Kil et al. (2013). Analysis of Serum Zinc and Copper Concentrations in Hair Loss. Ann Dermatol, 25.405-409.
[9] Gonzalez et al. (2010). Androgenetic Alopecia in The Paediatric Population: A Retrospective Review of 57 Patients. British J Dermatol, 163, 378-385.

[10] Ellis et al. (2010). Androgenetic Alopecia: Potential for Pathogenesis and Therapy. Expert Review, 1-12.

[11] Boldrin. (2010). Androgenic alopecia: Exploring Causes, Psychological Effects, with Western and Chinese Medicine Approach. Biomed Resc, 1-15.

[12] Malaysia Pd. (2003). Consensus statement on the management of anrogenic alopecia. Consensus.: 1-15.

[13] Skalnaya. (2014). Copper Deficiency A New Reason of Androgenetic Alopecia? Intech, 337-347. 